\title{
Simulación en laboratorio mediante ensayos de corrosión acelerada de la presencia y contenido en sales solubles en los productos de corrosión atmosférica del acero
}

\author{
D. de la Fuente*, B. Chico* y M. Morcillo* \\ Resumen En un intento de aproximación a lo que sucede en la atmósfera, en el presente trabajo, se \\ ha establecido una metodología mediante el empleo de ensayos de corrosión acelerada, \\ para contaminar superficies de acero con niveles de cloruros y sulfatos, del orden de los \\ encontrados de forma natural en la atmósfera. Los resultados obtenidos muestran que, \\ eligiendo como variable el tiempo de exposición en la cámara de niebla salina, en el caso \\ de los cloruros, y el volumen de $\mathrm{SO}_{2}$ introducido en la cámara $\mathrm{KESTERNICH}$, en el caso \\ de los sulfatos, ha sido posible contaminar substratos de acero con unos contenidos \\ preestablecidos en dichas sales, similares a los obtenidos de forma natural tras un período \\ de exposición de tres meses en tres atmósferas de distinta agresividad: Cabo Vilano \\ (marina), Avilés (industrial) y Madrid (urbana).
}

Palabras clave Sales solubles. Cloruros. Sulfatos. Ensayos de corrosión acelerada.

\section{Laboratory simulation using accelerated corrosion tests of soluble salt presence and content in the atmospheric corrosion products of steel}

\begin{abstract}
In an attempt to approximate what occurs in the atmosphere, this work has established a methodology using accelerated corrosion tests to contaminate steel surfaces with similar levels of chlorides and sulphates to those founded in atmospheric natural exposure. The results show that by selecting the variable of exposure time in the salt fog cabinet, in the case of chlorides, or the volume of $\mathrm{SO}_{2}$ introduced in the KESTERNICH cabinet, in the case of sulphates, it has been possible to contaminate steel substrates with similar preestablished contents of these salts to those obtained naturally after three months of exposure in three atmospheres of different aggressivities: Cabo Vilano (marine); Avilés (industrial); and Madrid (urban).
\end{abstract}

Keywords Soluble salts. Chlorides. Sulphates. Accelerated corrosion tests.

\section{INTRODUCCIÓN}

Es frecuente encontrar, entre los productos de corrosión atmosférica del acero, sales solubles, generalmente cloruros y sulfatos, cuando el acero ha estado expuesto durante un cierto tiempo en una atmósfera contaminada ${ }^{[1]}$. Los cloruros tienen su origen fundamentalmente en el aerosol marino, mientras que el $\mathrm{SO}_{2}$, procedente principalmente de la quema de combustibles fósiles, es el principal precursor de los sulfatos.

En la práctica, estas sales solubles se encuentran heterogéneamente distribuidas entre la herrumbre, acumulándose preferentemente en las capas más internas de los productos de corrosión ${ }^{[2}$ y 3]. Una primera causa que explica esta distribución es la acción de lavado que ejerce el agua de lluvia en las capas más externas de productos de corrosión, arrastrando las sales que se encuentran en estas capas y permitiendo su acumulación en las más internas. Además de este efecto lavador del agua de lluvia, una segunda causa que contribuye a la acumulación de las sales solubles en la interfase metal/capa de productos de corrosión es la migración hacia el interior de los iones. Esto se debe, según Evans ${ }^{[4]}$, a que en el acero, bajo la capa de productos de corrosión, se forman zonas anódicas de

(*) Centro Nacional de Investigaciones Metalúrgicas (CENIM) - CSIC, Avda. Gregorio del Amo 8, 28040 Madrid. Email: delafuente@cenim.csic.es 
micropilas de aireación diferencial, y los aniones, por procesos electroquímicos, migran hacia aquellas zonas, acumulándose.

En los primeros trabajos llevados a cabo por Mayne ${ }^{[5]}$ para estudiar el efecto negativo de las sales solubles, sobre el comportamiento posterior de los recubrimientos de pintura aplicados sobre substratos que contenían estas sales, se contaminaban las probetas de forma natural tras un período de exposición en una atmósfera determinada. Sin duda, se trata del método más fiel al caso real. Sin embargo, esta metodología presenta algunos inconvenientes, como la dificultad de obtener probetas contaminadas con niveles preestablecidos de sales solubles o los largos tiempos de exposición requeridos para conseguir contaminar las muestras con ciertos niveles.

Para solventar estos inconvenientes, era necesario desarrollar una metodología que permitiera obtener en el laboratorio, de forma sencilla, probetas contaminadas con diferentes niveles de sales solubles. En la mayor parte de los estudios de laboratorio realizados, que han tratado de simular esta condición superficial inicial del acero contaminado, se ha partido de substratos de acero limpios que, posteriormente, se han contaminado con los niveles deseados de sales solubles, dosificando y extendiendo con una varilla de vidrio la cantidad exacta de una determinada solución de dichas sales sobre la superficie metálica ${ }^{[6-8]}$. Aunque esta metodología ha sido ampliamente utilizada, se es consciente de que no representa fielmente la distribución real de las sales solubles que tiene lugar en la práctica, como ya apuntaron Bayliss y Chandler ${ }^{[9]}$.

De aquí, surge la necesidad de desarrollar nuevos métodos de laboratorio, para la obtención de muestras contaminadas con niveles preestablecidos de sales solubles y que supongan una condición intermedia entre la contaminación natural y la contaminación artificial mediante dosificación en laboratorio. De este modo, se podrían solventar los inconvenientes de la exposición natural (largos tiempos de exposición, niveles de contaminación inciertos, etc.) y obtener una distribución de las sales más cercana a la que tiene lugar en la atmósfera.

En un primer intento de aproximación a la situación real, se han llevado a cabo estudios, consistentes en dosificar cantidades conocidas de distintas sales sobre substratos de acero en los que se había generado con anterioridad una capa de herrumbre, y comparar los resultados con los obtenidos por el método tradicional de contaminación $^{[10]}$. Aunque se observó un cierto efecto sinérgico acelerante debido a la presencia conjunta de herrumbre y contaminantes, la distribución de las sales dosificadas sobre el substrato herrumbrado no es completamente fiel al caso real donde éstas van incorporándose paulatinamente a la capa de productos de corrosión.

En el presente trabajo, se ha llevado a cabo un nuevo intento de aproximación, estableciéndose una metodología mediante el empleo de ensayos de corrosión acelerada. De este modo, ha sido posible contaminar substratos de acero con unos contenidos de cloruros y sulfatos, similares a los obtenidos de forma natural tras un período de exposición de tres meses en tres atmósferas de distinta agresividad, Cabo Vilano (marina), Avilés (industrial) y Madrid (urbana).

\section{MATERIALES Y PROCEDIMIENTO EXPERIMEN- TAL}

Series de probetas de acero EN 10025 de $100 \times$ $150 \times 5 \mathrm{~mm}$, chorreadas con granalla angular de acero G17/G24 hasta el grado ASa3 según la norma sueca SIS $055900^{[11]}$ y con un perfil de rugosidad de $75 \mu \mathrm{m}$, se expusieron en tres atmósferas de diferente agresividad: Cabo Vilano (marina), Avilés (industrial) y Madrid (urbana) (tabla I). Después de tres meses, fueron retiradas y almacenadas en bolsas de plástico con cierre hermético.

Tabla I. Características de las estaciones de ensayo

Table I. Characteristics of the exposure test sites

\begin{tabular}{lccc}
\hline Estación de Ensayo & Depósito de $\mathbf{C l},{\mathbf{~ m g} \cdot \mathbf{m}^{-2} \mathbf{d}^{-1}}$ & Depósito de $\mathbf{S O}_{2}, \mathbf{m g} \cdot \mathbf{m}^{-2} \mathbf{d}^{-1}$ & Corrosividad ISO \\
\hline Cabo Vilano & 921,27 & 60,80 & $\mathrm{C} 5$ \\
Avilés & 66,6 & 19,2 & $\mathrm{C} 4$ \\
Madrid & 0,9 & 36,6 & $\mathrm{C} 2$ \\
\hline
\end{tabular}


Simulación en laboratorio mediante ensayos de corrosión acelerada de la presencia y contenido en sales solubles en los productos de corrosión atmosférica del acero

D. DE LA Fuente, B. Chico y M. MORCILlo

\subsection{Extracción y análisis del contenido en clo- ruros y sulfatos}

Se emplearon tres probetas por cada estación de ensayo para determinar la cantidad de cloruros o sulfatos presentes, previa extracción de los mismos. Otras dos series de tres probetas por estación se limpiaron mediante cepillado automático y chorreado ligero con arena respectivamente, para analizar también el contenido residual en ambas sales tras ambos métodos de limpieza. Para realizar la extracción se utilizó el método convencional de laboratorio, conocido como método Mayne ${ }^{[5]}$. Consiste en la introducción de las muestras, por separado, en agua destilada a ebullición durante 60 min. A continuación, se filtran las soluciones y se llevan a volumen constante para su posterior análisis.

Los iones cloruro en solución se analizaron mediante un electrodo selectivo de cloruros.

Los iones sulfato, se analizaron mediante el método turbidimétrico, norma ASTM D516 ${ }^{[12]}$. El sulfato en solución es transformado a sulfato de bario en suspensión mediante la adición de cloruro de bario en condiciones controladas. Mediante un espectrofotómetro se mide la absorbancia de la solución en el rango del ultravioleta que es lineal con la concentración inicial de ion sulfato en la solución.

\subsection{Simulación en laboratorio}

Con el fin de obtener probetas contaminadas artificialmente con niveles y distribución de estos dos contaminantes, similares a los obtenidos de manera natural en las tres atmósferas, se ha intentado reproducir en el laboratorio, en una cámara de niebla salina para el caso de los cloruros y en una cámara tipo KESTERNICH para los sulfatos, el proceso de corrosión atmosférica del acero en ambientes contaminados con cloruros y $\mathrm{SO}_{2}$.

\section{RESULTADOS Y DISCUSIÓN}

En la figura 1 se presentan los niveles obtenidos de cloruros después de tres meses de exposición en cada estación de ensayo, tanto sin limpieza posterior como tras la aplicación de un cepillado automático o un chorreado ligero con arena. Pueden observarse dos diferentes comportamientos: el primero, correspondiente a las atmósferas no marinas $(\approx 3,5$ $4,0 \mu \mathrm{g} \mathrm{Cl} / \mathrm{cm}^{2}$ de contenido total y 3,0-3,5 $\mu \mathrm{g}$ $\mathrm{Cl}^{-} / \mathrm{cm}^{2}$ de contenido residual tras el chorreado li-

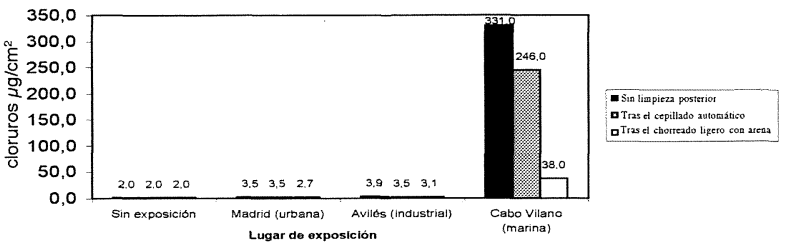

Figura 1. Niveles de cloruros tras tres meses de exposición en la atmósfera.

Figure 1. Chlorides levels after three months of natural exposure.

gero con arena) y, el segundo, correspondiente a la atmósfera marina $\left(\approx 330 \mu \mathrm{g} \mathrm{Cl} / \mathrm{cm}^{2}\right.$ de contenido total y $35-40 \mu \mathrm{g} / \mathrm{cm}^{2}$ de contenido residual tras el chorreado ligero). De acuerdo con la experiencia previa $^{[13}$ y 14$]$ para la obtención de muestras contaminadas con diferentes niveles de cloruros en la cámara de niebla salina, la variable más adecuada es el tiempo de exposición de las muestras en la cámara. En la figura 2 se presenta la relación entre el contenido de cloruros total, antes de aplicar cualquier procedimiento de limpieza y el tiempo de exposición en la cámara.

De acuerdo con la figura 2, para obtener el nivel similar al obtenido en la atmósfera marina $\left(\approx 330 \mu \mathrm{g} \mathrm{Cl} / \mathrm{cm}^{2}\right)$, las muestras se expusieron en la cámara de niebla salina durante $30 \mathrm{~h}$. En el caso de la simulación de las atmósferas no marinas se expusieron durante $10 \mathrm{~min}$. En la tabla II se muestran los resultados obtenidos.

Como puede observarse, en el caso de la simulación de las atmósferas no marinas, el nivel de cloruros medido después del chorreado con arena $\left(5 \mu \mathrm{g} \mathrm{Cl} / \mathrm{cm}^{2}\right)$ es muy similar al nivel deseado que se

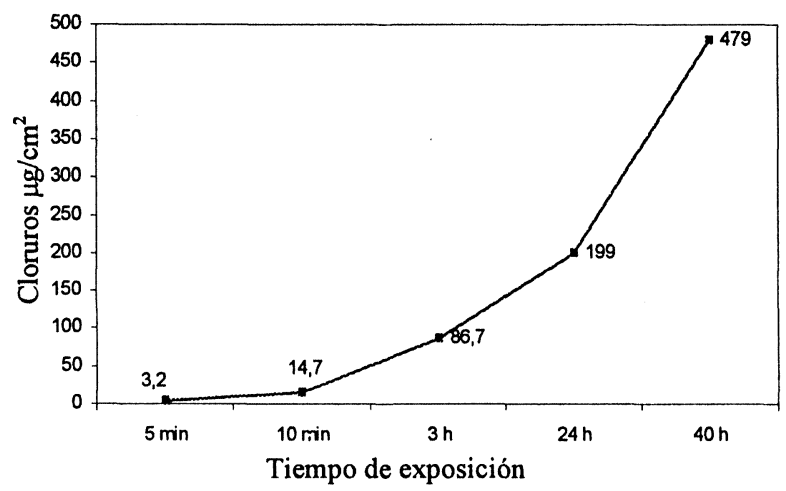

Figura 2. Nivel de cloruros, antes de la limpieza, en función del tiempo de exposición en la cámara de niebla salina.

Figure 2. Chlorides level, before cleaning, versus exposure time in the salt fog cabinet.

Rev. Metal. Madrid Vol. Extr. (2005) 438-442 
Tabla II. Cloruros obtenidos antes y después del chorreado con arena

Table II. Chlorides measured before and after the sand blast cleaning

\begin{tabular}{|c|c|c|c|c|c|}
\hline $\begin{array}{l}\text { Simulación } \\
\text { de las } \\
\text { atmósferas }\end{array}$ & $\begin{array}{c}\text { Tiempo de } \\
\text { exposición } \\
\text { en cámara de } \\
\text { niebla salina }\end{array}$ & $\begin{array}{c}\text { Nivel deseado } \\
\text { antes del } \\
\text { chorreado ligero, } \\
\mu \mathrm{g} / \mathrm{cm}^{2}\end{array}$ & $\begin{array}{c}\text { Nivel deseado } \\
\text { después del } \\
\text { chorreado ligero, } \\
\mu \mathrm{g} / \mathrm{cm}^{2}\end{array}$ & $\begin{array}{c}\text { Nivel medido } \\
\text { antes del } \\
\text { chorreado ligero, } \\
\mu \mathrm{g} / \mathrm{cm}^{2}\end{array}$ & $\begin{array}{c}\text { Nivel medido } \\
\text { después del } \\
\text { chorreado ligero, } \\
\mu \mathrm{g} / \mathrm{cm}^{2}\end{array}$ \\
\hline No marinas & $10 \mathrm{~min}$ & $3,5-4$ & $3-3,5$ & 14,7 & 5 \\
\hline Marina & $30 \mathrm{~h}$ & 330 & $35-40$ & 453 & 20,7 \\
\hline
\end{tabular}

había obtenido en la atmósfera $\left(3-3,5 \mu \mathrm{g} \mathrm{Cl} / \mathrm{cm}^{2}\right)$. Sin embargo, en el caso de la simulación de la atmósfera marina después del chorreado, el nivel de cloruros obtenido $\left(20,7 \mu \mathrm{g} \mathrm{Cl}^{-} / \mathrm{cm}^{2}\right)$ es mucho menor que el esperado $\left(35-40 \mu \mathrm{g} \mathrm{Cl}^{-} / \mathrm{cm}^{2}\right)$, aún cuando antes de la limpieza, se había obtenido un mayor nivel $\left(453 \mu \mathrm{g} \mathrm{Cl} / \mathrm{cm}^{2}\right)$ del pretendido $\left(330 \mu \mathrm{g} \mathrm{Cl} / \mathrm{cm}^{2}\right)$. Este resultado podría explicarse porque, en el caso de la contaminación natural, los cloruros están situados en las capas más internas de los productos de corrosión mientras que, en el caso de la contaminación en la cámara, los cloruros no tienen tiempo suficiente para alcanzar estas capas internas y estarían situados más cerca de la superficie, donde son eliminados más fácilmente durante el chorreado. Para solventar esta dificultad, en un primer intento para obtener un mayor nivel de cloruros después del chorreado, se intentó aumentar el tiempo de exposición en la cámara de niebla salina. Sin embargo, aunque el nivel de cloruros total, obtenido antes de la limpieza, aumentó bastante con el tiempo de exposición, la cantidad de cloruros residual tras el chorreado sólo aumento muy ligeramente, probablemente por la misma razón explicada anteriormente de la distribución de los cloruros en las capas más externas. En un segundo intento de aproximación, se expusieron las muestras en la cámara de condensación de humedad, tras la exposición en la cámara de niebla salina, con la intención de permitir que los cloruros tuvieran tiempo de alcanzar las capas más internas. Sin embargo, el resultado obtenido fue opuesto al esperado y se midieron niveles de cloruros tras el chorreado, menores que sin el empleo de la cámara de condensación, probablemente debido a un efecto lavador del agua. Finalmente, se optó por aplicar un chorreado más ligero, reduciendo la presión y el tiempo de limpieza, sobre las muestras expuestas durante $30 \mathrm{~h}$ en la cámara de niebla salina (453 $\mu \mathrm{g} \mathrm{Cl} / \mathrm{cm}^{2}$ antes del chorreado). De este mo- do, la cantidad residual de cloruros obtenida tras el chorreado ligero ( $\left.35 \mu \mathrm{g} \mathrm{Cl} / \mathrm{cm}^{2}\right)$ resultó muy próxima a la que se había obtenido en la atmósfera marina de Cabo Vilano $\left(38 \mu \mathrm{g} \mathrm{Cl} / \mathrm{cm}^{2}\right)$.

En el caso de los sulfatos también pueden definirse dos niveles (Fig. 3), el de la atmósfera industrial y el de las atmósferas no industriales.

Conforme a la experiencia previa ${ }^{[13}$ y 14$]$, para obtener superficies contaminadas con diferentes niveles de sulfatos en la cámara KESTERNICH, la variable más apropiada es el volumen de $\mathrm{SO}_{2}$ introducido, fijando el tiempo de exposición en 2,5 h y la superficie total expuesta en 46 probetas $\left(1,495 \mathrm{~m}^{2}\right)$. En la figura 4 se presenta la relación obtenida en nuestro caso.

De acuerdo con la relación mostrada en la figura 4, para obtener un nivel de sulfatos similar al obtenido en la atmósfera industrial $(39,6 \mu \mathrm{g}$ $\mathrm{Cl}^{-} / \mathrm{cm}^{2}$ y $25,7 \mu \mathrm{g} \mathrm{Cl} / \mathrm{cm}^{2}$, antes y después del chorreado, respectivamente), se introdujo en el interior de la cámara un volumen de 0,5 1 de $\mathrm{SO}_{2}$. En el caso de las atmósferas no industriales, el volumen introducido fue de 0,2 1 de $\mathrm{SO}_{2}$. En ambos casos, los niveles obtenidos tanto antes como después del chorreado, fueron muy próximos a los obtenidos de manera natural.

En resumen, se han obtenido en las cámaras, superficies de acero contaminadas con tres niveles

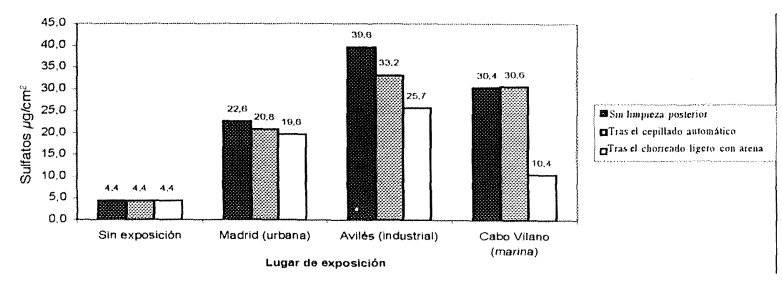

Figura 3. Niveles de sulfatos tras tres meses de exposición en la atmósfera.

Figure 3. Sulphates levels after three months of natural exposure 
Simulación en laboratorio mediante ensayos de corrosión acelerada de la presencia y contenido en sales solubles en los productos de corrosión atmosférica del acero

D. DE lA Fuente, B. ChiCO y M. MORCILlo

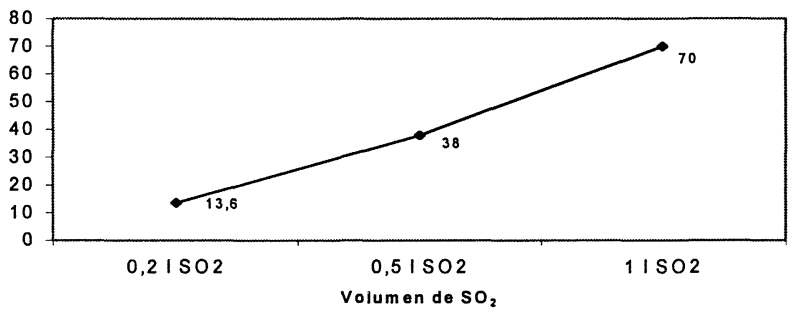

Figura 4. Nivel de sulfatos, antes de la limpieza, en función del volumen de $\mathrm{SO}_{2}$ introducido en la cámara KESTER$\mathrm{NICH}$.

Figure 4. Sulphates level, before cleaning, in relation to the $\mathrm{SO}_{2}$ volume injected in the KESTERNICH cabinet.

diferentes de cloruros-sulfatos: a) simulando la atmósfera marina de Cabo Vilano ( $30 \mathrm{~h}$ de niebla salina $+2,5 \mathrm{~h}$ en cámara KESTERNICH con 0,21 de $\mathrm{SO}_{2}$ ); b) simulando la atmósfera industrial de Avilés (10 min de niebla salina $+2,5$ h en cámara KESTERNICH con 0,5 1 de $\mathrm{SO}_{2}$ ); y c) simulando la atmósfera urbana de Madrid (10 min de niebla salina $+2,5 \mathrm{~h}$ en cámara KESTERNICH con 0,21 de $\mathrm{SO}_{2}$ ).

\section{CONCLUSIONES}

- Se ha establecido una metodología para obtener, mediante ensayos de corrosión acelerada en cámaras, superficies de acero contaminadas con niveles preestablecidos de cloruros/sulfatos, similares a los obtenidos de manera natural y con una distribución de los mismos más fiel a la que ocurre en la atmósfera que la obtenida con los métodos convencionales de dosificación homogénea.

- En el caso de los cloruros, la variable elegida ha sido el tiempo de exposición en la cámara de niebla salina, mientras que en el caso de los sul- fatos ha sido el volumen de $\mathrm{SO}_{2}$ introducido en la cámara KESTERNICH.

\section{Agradecimientos}

Los autores del presente trabajo desean mostrar su agradecimiento a ARCELOR y la Universidad de Vigo por la cesión de las estaciones de ensayo de corrosión atmosférica utilizadas para la realización de este estudio.

\section{REFERENCIAS}

[1] M. Morcillo, Rev. Metal. Madrid, Vol. Extr. (2003) 219. 227.

[2] J.B. Harrison y T.C.K. Tickle, J. Oil. Colour Chem. Assoc. 45 (1962) 571-597.

[3] S. Flores y M. Morcillo, Surf. Coat. Int. 82 (1999) 19. 25.

[4] U.R. Evans, Nature 206 (1965) 1.980.

[5] J.E.O. MAYNE, J. Appl. Chem. 9 (1959) 673-680.

[6] M. Morcillo, S. Feliu, J.C. Galván y J.M. Bastidas, J. Oil. Colour Chem. Assoc. 71 (1988) 11-16.

[7] M. Morcillo y J. Simancas, J. Prot. Coat. Linings 14 (1997) 40-52.

[8] H. MitschKe, J. Prot. Coat. Linings 18 (2001) 49.

[9] D.A. BAYliss y K.A. ChANDLER, Steelwork Corrosion Control, Ed. Elsevier, Reino Unido, 1991, p.28.

[10] M. Morcillo, S. Feliu y J. Simancas, Farbe + Lack 95 (1989) 726-728.

[11] SIS 055900, Swedish Standard Institute, Stockholm, 1967.

[12] ASTM D-516, 1968.

[13] D. De La Fuente, B. Chico y M. Morcillo, Rev. Metal. Madrid, Vol. Extr. (2003) 129-136.

[14] D. De La Fuente, B. Chico y M. Morcillo, Anti-Corros. Methods Mater. 50 (2003) 208-216. 